\title{
Selective Reinnervation of Transplanted Muscles by their Original Motoneurons in the Axolotl
}

\author{
Donald J. Wigston and Philip R. Kennedy ${ }^{a}$ \\ Department of Physiology, Emory University School of Medicine, Atlanta, Georgia 30322
}

The motoneurons innervating 3 hindlimb extensor muscles, anterior and posterior iliotibialis and iliofibularis, were studied separately by retrograde labeling with HRP. The motor pools for these 3 muscles overlapped to such an extent that individual motoneurons between ventral roots 16 and 17 could not be assigned unambiguously to one pool or another. Thus, conventional retrograde labeling could not identify particular axolotl motoneurons. Instead, a double retrograde-labeling technique was employed to mark the motoneurons innervating a particular muscle, the left posterior iliotibialis. Either diamidino yellow (DY) or HRP satisfactorily labeled axolotl motoneurons for at least 3 months in vivo. After labeling, both anterior and posterior iliotibialis muscles were removed from the injected limb and replaced with their counterparts from the opposite limb, in reversed anteriorposterior orientation. Several weeks later, a second marker (DY or HRP) injected into the posterior iliotibialis muscle in its new, more anterior, position labeled the neurons that reinnervated this muscle; the number of neurons labeled with both first and second tracers gave an indication of the selectivity of reinnervation. Using this approach, we have found that the majority of neurons reinnervating a particular muscle are members of that muscle's original motor pool.

The remarkable ability of adult urodele amphibians to regain normal motor function after nerve damage offers a splendid opportunity to study the factors that control synaptic specificity. There is evidence that 3 quite different mechanisms might contribute to the selectivity of motor nerve regeneration in urodeles. First, it appears that even in this nonembryonic setting, regenerating motor axons are capable of navigating to their proper target muscles (Grimm, 1971; Holder et al., 1984; D. J. Wigston, unpublished observations). Second, axons seem to be capable of distinguishing between different muscles such that the restoration of connections between appropriate pre- and postsynaptic partners is favored (Stephenson, 1979; Holder et al., 1982; Wigston, 1986). Third, synaptic connections between inappropriate synaptic partners may be eliminated competitively in favor of better matched connections (Bennett and Raftos, 1977; Dennis and Yip, 1978). This report is concerned with the second

\footnotetext{
Received Sept. 5, 1986; revised Dec. 8, 1986; accepted Dec. 15, 1986.

We gratefully acknowledge L. Crews for helping with some of the dye injections; N. Marshall for carrying out the electrophysiology; P. Shuler for technical assistance; A. McLean for help in preparing the manuscript; and A. English, D. Purves, and $J$. Sanes for helpful suggestions and criticism. This work was supported by a BRSG grant to Emory University and NIH Grant NS20965 to D.W.

Correspondence should be addressed to Donald J. Wigston at the above address.

a Present address: Georgia Tech. Research Institute, Atlanta, GA 30332.

Copyright (C) 1987 Society for Neuroscience $0270-6474 / 87 / 061857-09 \$ 02.00 / 0$
}

of these mechanisms: the selectivity of synapse formation itself.

Earlier work that examined selective synapse formation relied primarily on electrophysiological methods-recording from individual muscle fibers and stimulating spinal or muscle nervesto determine the segmental origin or the quantal content of detected inputs to a particular fiber. For instance, nerves could be rerouted to innervate foreign muscles, and the strength of the synapses they made could be determined by measuring their quantal content; this could then be compared with the strength of transmission at synapses regenerated in the nerve's original muscle (Holder et al., 1982). More recently, muscles have been denervated, transplanted to new positions, and their reinnervation assessed by stimulating spinal nerves (Wigston, 1986). These experiments have shown that individual muscles tend to be reinnervated by axons in the same spinal nerves as before, and in the proper proportions, even after transplantation to a novel site. Thus different axolot limb muscles are reinnervated selectively.

The experiments reported here were designed to examine the selectivity of muscle reinnervation from the vantage point of individual motoneurons, rather than of spinal cord regions (segmental nerves) or groups of neurons (muscle nerves). For example, they test whether muscles are indeed reinnervated by the same motoneurons that innervated them originally or, instead, are innervated by any neurons that send their axons into the same spinal nerve. Since axolotl motoneuron pools extend over large distances, so that the rostrocaudal positions of different motor pools overlap extensively (Székely and Czéh, 1967; Stephens and Holder, 1985), earlier results might reflect a general correspondence between positionally appropriate motoneurons and particular muscles, rather than a true restoration of original connections. A double retrograde-labeling technique has made it possible to examine this question directly. Some of this work has been presented previously (Wigston and Kennedy, 1986).

\section{Materials and Methods}

Animals. Male and female wild-type axolotls (Ambystoma mexicanum; $10-25 \mathrm{~cm}$ ) were obtained from Central Valley Biological (Gardnerville, NV). They were housed individually in trays of $20 \%$ modified Holtfreter's solution at $20 \pm 2^{\circ} \mathrm{C}$, and fed beef liver or live worms ( $\mathrm{Lum}$ briculus) twice weekly.

Tracer injections. Animals were anesthetized in $0.1 \% \mathrm{MS} 222$ (tricaine methanesulfonate; Sigma) for $20-30 \mathrm{~min}$, laid in an operating dish, and covered with moist tissue. The appropriate muscle was exposed (see Fig. 1) and pressure-injected with tracer from a glass micropipette (tip diameter, $\approx 50 \mu \mathrm{m}$ ). HRP (Sigma Type VI) was injected as either a $40 \%$ solution in distilled water or a $2-10 \%$ solution of HRP covalently linked to wheat-germ agglutinin (WGA-HRP; Sigma). Fast green was added to both types of HRP solution to help visualize the initial extent of the injection site. Diamidino yellow dihydrochloride(DY; Dr. Illing, GmbH 


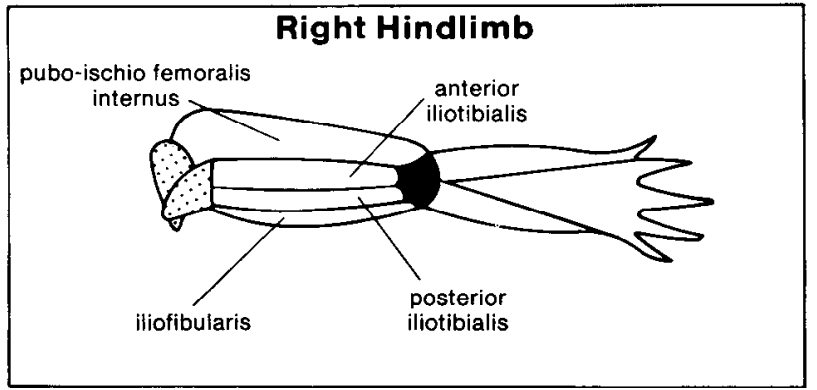

Figure 1. Diagram of the dorsal surface of the right hindlimb of an axolotl. The outlines of the 7 main dorsal muscles are shown and the four proximal muscles labeled. Anterior is up, posterior down.

and Co., FDR) was injected as a $2 \%$ suspension in distilled water. Several (usually 5-7) injections of $0.01-0.1 \mu \mathrm{l}$ each were made along the length of the muscle; this was preferred to a single, larger injection, since axolotl muscle fibers are innervated at several points (Lehouelleur and Chatelain, 1974; Slack and Docherty, 1978). Adjacent muscles were covered with wafers of moist paper tissue to protect them from spilled tracer and to prevent dehydration. Any spilled dye or enzyme was immediately wiped away and the affected area irrigated with Ringer's solution. Control experiments showed that deliberate applications of DY or HRP to muscle surfaces did not label any motoneurons. For transplantation experiments, DY or WGA-HRP (5 or $10 \%$ ) was injected into the left postcrior iliotibialis (ILT) $5-11 \mathrm{~d}$ before surgery.

Surgery. Muscle transplantations were performed as described previously (Wigston, 1986). Briefly, the anterior and the injected posterior ILT muscles in the left hindlimb were replaced by their counlerparts from the right hindlimb, in reversed anterior-posterior orientation (see Fig. 3). In this way, an anterior ILT replaced a posterior ILT, while a posterior ILT occupied the site vacated by an anterior ILT. The left anterior and posterior ILT muscles were sutured into the right hindlimb. Both ILT muscles were denervated by this procedure, since during their removal, the several small nerve branches that provide their innervation were severed. During regeneration, all severed axons probably have equal access to both anterior and posterior muscles (Wigston, 1986).

Fixation. After total survival times of 3-5 d for acute labeling experiments, or 57-89 d for reinnervation experiments, animals were anesthetized and perfuscd through the heart with saline $(10-100 \mathrm{ml}$; $0.9 \%)$ followed by fixative $(10-100 \mathrm{ml} ; 2.5 \%$ glutaraldehyde, $0.1 \mathrm{M}$ phosphate buffer, and $4 \%$ sucrose or, for fluorescently labeled animals, $4 \%$ paraformaldehyde, $0.2 \%$ glutaraldehyde, $0.1 \mathrm{~m}$ citrate buffer, $4 \%$ sucrose, $\mathrm{pH}$ 7.2). Immediately after perfusion, isolated lumbar regions were immersed in fixative and the spinal cord exposed by a ventral laminectomy. The spinal cord from nerves 14 to 19 was removed, stripped of meninges, pinned in a dish, and immersed in fixative containing $20 \%$ sucrose for a further $3 \mathrm{hr}$ at $4^{\circ} \mathrm{C}$. After rinsing in 2 changes of $20 \%$ buffered sucrose for $1 \mathrm{hr}$ or overnight at $4^{\circ} \mathrm{C}$, cords were trimmed to include segments $15-18$ (usually 10-15 mm long) and embedded in gelatin-albumin. Iliofibularis and anterior and posterior II.T muscles were removed from both hindlimbs, fixed, and embedded in a similar way. The gelatin blocks containing the muscle or spinal cord were fixed at $4^{\circ} \mathrm{C}$ for either $1 \mathrm{hr}$ in $2.5 \%$ glutaraldehyde fixative or $3 \mathrm{hr}$ in $4 \%$ paraformaldehyde fixative.

Tissue processing. Gelatin-embedded tissue was frozen with dry ice, and transverse sections of spinal cord or horizontal sections of inuscle cut on a sliding microtome at $50 \mu \mathrm{m}$. To demonstrate HRP, the sections were reacted using 3,3',5,5'-tetramethylbenzidine (TMB) as the chromogen (modified from Mesulam, 1978). In brief, sections were equilibrated

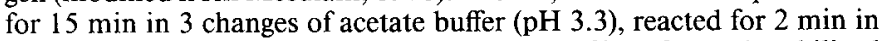
$0.3 \mathrm{~mm}$ acetate-buffered TMB containing $0.03 \% \mathrm{H}_{2} \mathrm{O}_{2}$, and stabilized for $2 \mathrm{~min}$ in acetate-buffered $0.1 \%$ sodium nitroferricyanide that also contained $0.03 \% \mathrm{H}_{2} \mathrm{O}_{2}$ before being returned to acetate buffer for a further $2 \mathrm{~min}$. At this point, sample sections were examined with a microscope and the appearance of labeled cells noted. The reaction cycle was repeated (usually 1-2 times) until satisfactory labeling was achieved. Sections were then mounted on gclatinized slides, dried overnight, dehydrated in a graded series of acetone, and coverslips were mounted with DPX (Gallard-Schlesenger). Sections labeled with HRP only were lightly counterstained with neutral red; otherwise, no counterstaining was employed.
Analysis. Each section was examined with bright-field illumination; fluorescently labeled specimens were also examined with epi-illumination (excitation, $340-380 \mathrm{~nm}$; Leitz filter cube A), using a $50 \times$ objective (N.A. = 1.00). Photomicrographs were made with Kodak Tri-X film at ASA 400 or with Kodak Technical Pan at ASA 100. All but 8 experiments were analyzed independently by both P.R.K. and D.J.W., usually within $48 \mathrm{hr}$ of processing, and the 2 counts averaged; variation between counts was usually less than $10 \%$. Only clearly labeled cells were included in the analysis. Obvious fragments of cells were not included, but otherwise no correction was applied for double counting of split cells. Our counts, then, may overestimate the true numbers by as much as $30 \%$ (see Abercrombie, 1946). The position and extent of ventral roots $15-18$ were noted on the tally sheets to establish segmental boundaries. To construct the histograms in Figure 2, each spinal cord was divided into groups of sections representing $5 \%$ of the total distance between the middle of ventral roots 15 and 18. The number of cells appearing in each 5\% group was expressed as a percentage of the total number of cells labcled in that motor pool. The mean of these values for all spinal cords from each group of animals was then calculated for each $5 \%$ block and plotted on the histogram. In this way, results from animals that differed in their overall length and degree of labeling could be brought into register and displayed in a single histogram (see Landmesser, 1978). No attempt was made to eliminate the differences between animals in overall rostrocaudal position of the hindlimb motoneurons relative to the position of the ventral roots (Bennett and Raftos, 1977; Stephens and Holder, 1985; Wigston, 1986). For double-label experiments, the number of cells labeled with both tracers was expressed as a percentage of the total number labeled with the second tracer injected. Experiments were rejected if muscles adjacent to the one injected showed heavy cross-labeling. Except for an earlier series of HRPinjected animals (not included), this rarely occurred; a single experimental case was rejected because the intensity of labcling in anterior and posterior muscles was identical.

Electrophysiology. To test the possibility that motor axons branched to more than 1 muscle, we searched for axon reflexes using conventional extracellular recording and stimulation techniques. In brief, hindlimbs were isolated and prepared for recording as described previously (Wigston, 1986). In most experiments, the anterior and posterior ILT muscles were removed and small nerve branches to each muscle identified. Closefitting glass suction electrodes were used to stimulate spinal nerves $15-$ 18 separately, and others, made from finely tapered glass micropipettes, were used to stimulate and record from the proximal cut ends of individual muscle nerves. Four separate nerve branches could normally be isolated for each muscle. Each branch was stimulated repeatedly at $0.5 \mathrm{~Hz}$ ( $10 \mathrm{~V}$ pulses, $50 \mu \mathrm{sec}$ duration) while recording in turn from the 4 branches innervating the adjacent musclc. In all, 16 combinations of stimulation and recording from anterior and posterior ILT nerve branches were possible. Spinal nerves $15-18$ were stimulated periodically throughout this procedure, and the responses in muscle nerve branches were checked; these did not change appreciably over several hours.

\section{Results}

One way to identify the neurons that innervate a particular target is to inject the target with a tracer, such as HRP, that is retrogradely transported and labels the neuronal cell bodies (Lamb, 1976; Hollyday et al., 1977; Brushart and Mesulam, 1980). In some species, the motoneurons normally innervating a particular muscle are tightly clustered in the spinal cord, and hence the identity of labeled cells can be reliably predicted from their unique position (Landmesser, 1978; Hollyday, 1980; LanccJones and Landmesser, 1980). We therefore first set out to characterize the number and position of motoneurons innervating 3 hindlimb muscles, the anterior iliotibialis, posterior iliotibialis, and iliofibularis, using retrogradely transported markers.

\section{Retrograde labeling of hindlimb motor pools}

Number and distribution of normal motor pools. We injected anterior ILT muscles in 6 animals with WGA-HRP; on average, $30 \pm 5$ neurons (uncorrected for split cells) were labeled 4-5 d later (Table 1). We injected posterior ILT with WGA-HRP, 
Table 1. Number of motoneurons labeled by retrograde transport ${ }^{a}$

\begin{tabular}{llcl} 
Muscle injected & Tracer & $\begin{array}{l}\text { Transport } \\
\text { time } \\
\text { (d) }\end{array}$ & $\begin{array}{l}\text { No. of labeled } \\
\text { neurons }\end{array}$ \\
\hline Antcrior iliotibialis & HRP & $4-5$ & $30 \pm 5(6)^{b}$ \\
Posterior iliotibialis & HRP & $4-5$ & $42 \pm 4(4)$ \\
& HRP & $56-70$ & $35 \pm 6(8)$ \\
& DY & $9-11$ & $46 \pm 8(6)$ \\
Iliofibularis & DY & $65-88$ & $89 \pm 13(14)$ \\
& HRP & $4-5$ & $13 \pm 2(4)$ \\
\hline
\end{tabular}

${ }^{a}$ Uncorrected for split cells.

${ }^{b}$ Mean $\pm \mathrm{SE}(n)$. For posterior ILT, significance of differences (Student's $t$ test) was as follows: HRP (4-5 d) and DY $(9-11 \mathrm{~d}), p<0.7$; HRP (4-5 d) and HRP $(56-70 \mathrm{~d}), p<0.4$; DY $(9-11 \mathrm{~d})$ and DY $(65-88 \mathrm{~d}), p<0.02$.

labeling $42 \pm 4(n=4)$ neurons, whereas $13 \pm 2(n=4)$ neurons were labeled after injecting iliofibularis.

For mapping the distributions of labeled cells, 3 animals were injected with WGA-HRP in the anterior ILT on one side, and in the posterior ILT on the other. Two additional animals received HRP or WGA-HRP injections to the iliofibularis on both sides. The results, normalized as described in Materials and Methods, are shown in Figure 2. Labeled cells in spinal cord cross-sections were counted after allowing 4-5 d for retrograde transport (see Table 1). The rostrocaudal positions of all 3 motor pools overlapped considerably (Fig. 2), as did their mediolateral and dorsoventral positions (see Székely and Czéh, 1967). Indeed, it would be impossible to assign labeled neurons that were located between the middle of ventral roots 16 and 17 to their proper motor pool on the basis of their positions alone. In spite of this, the location of the motoneurons did shift somewhat for each motor pool: the median of each distribution, indicated by the black triangles in Figure 2, shifted progressively towards more caudal positions from (Fig. $2 a$ ) anterior ILT through (Fig. $2 b$ ) posterior ILT to (Fig. $2 c$ ) iliofibularis. This shift is consistent with the electrophysiological data from the same muscles obtained by intracellular recording and spinal nerve stimulation (Wigston, 1986). However, the limited ability to identify a motoneuron from its position within the spinal cord ruled out straightforward retrograde labeling as a way of identifying the motoneurons reinnervating a muscle.

\section{Double retrograde labeling of the neurons innervating and reinnervating a muscle}

Combined DY/HRP labeling. Some retrograde neuronal tracers persist within living neurons for long enough for us to perform reinnervation experiments (see Bentivoglio et al., 1980; O'Leary et al., 1981; Sawchenko and Swanson, 1981). We chose diamidino yellow dihydrochloride (DY) because it has been reported to (1) remain inside cells for some time, (2) leak only very slowly out of labeled cells, and (3) be compatible with histochemical processing to demonstrate HRP (Huisman et al., 1983; Cavada et al., 1984). Experiments in which we injected posterior ILT muscles with $2 \%$ DY showed that 9-11 d later, a similar number of neurons ( $46 \pm 8, n=6$ ) were labeled as were labeled after WGA-HRP injection ( $42 \pm 4, n=4$; Table 1$)$. Thus, DY is an efficient retrograde tracer, comparable to HRP. Further experiments revealed that motoneurons were indeed still clearly labeled up to 3 months after intramuscular injection of DY.

Our strategy was to mark the motoneurons innervating a sin-

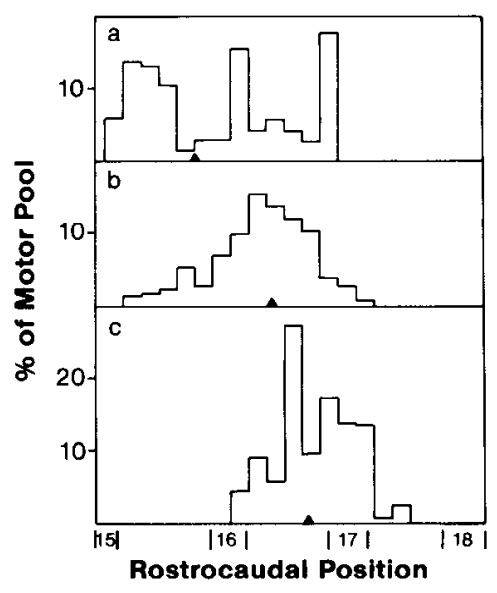

Figure 2. The distribution of motoneurons labeled by HRP injections into hindlimb muscles. $a$. Anterior ILT $(n=6) . b$. Posterior ILT $(n=$ 4). $c$, Iliofibularis $(n=4)$. Distributions were normalized as described in Materials and Methods and show the percentage of the total labeled motor pool found within each $5 \%$ of the cross sections obtained between the middle of ventral roots 15 and 18; black triangles represent the median for each distribution.

gle muscle, usually the left posterior ILT, by injecting the muscle with DY. Nine to 11 d later, after retrograde transport of the dye into motoneuronal cell bodies, we exchanged the positions of anterior and posterior ILT muscles by removing them together from the right limb and suturing them in reversed anterior-posterior orientation into the left limb (see Fig. 3). The result of this manipulation was that the posterior ILT occupied the normal position of an anterior ILT, while the anterior ILT assumed the site vacated by the posterior ILT. Both muscles were denervated at the point of entry of their nerves. After waiting 6-10 weeks for reinnervation of the transplanted muscles, we injected HRP or WGA-HRP into the original posterior ILT in its novel, more anterior position. Following uptake of the second tracer, we examined neurons in spinal cord sections with both epifluorescence and bright-field optics. Neurons that contained both tracers had a yellow DY-labeled nucleus surrounded by blue/black cytoplasmic HRP reaction product (Fig. 4). Such doubly labeled cells are likely to be motoneurons that originally innervated the posterior ILT and successfully reinnervated it after transplantation. We expressed the degree of double-labeling as the percentage of neurons labeled with peroxidase that also had a yellow, DY-labeled nucleus. This probably provides the most valid indication of the selectivity of reinnervation. The number of neurons labeled with HRP should reflect the number of neurons that innervated the transplanted posterior ILT. These could be neurons that originally innervated posterior ILT, anterior ILT, or perhaps other muscles. Therefore, the proportion of HRP-labeled cells that was also labeled with DY should give the proportion of neurons reinnervating the posterior ILT that were original members of the posterior ILT motoneuron pool. An alternative would be to express the number of HRP-labeled cells as a percentage of DY-labeled cells; however, this fails to allow for posterior ILT motoneurons that might not have regenerated and therefore would have no chance of reinnervating posterior ILT and taking up HRP. Indeed, the number of neurons labeled with HRP by the second injection was consistently smaller than the number still labeled by the initial DY injection (however, sce bclow). Sincc we were interested in the selectivity, rather than the efficiency, of rein- 


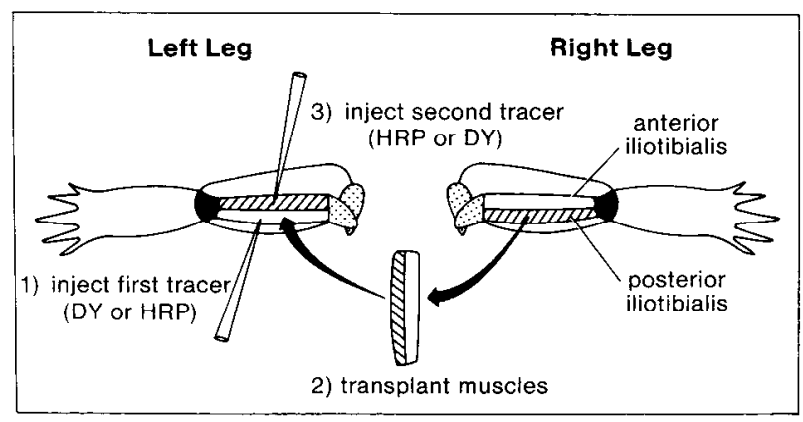

Figure 3. Diagram of retrograde tracer injection protocol. 1, The first tracer was injected into the left posterior ILT as described in Materials and Methods. 2, After 5-10 d, the left anterior and posterior ILT muscles were replaced by the same pair of muscles from the right side, but in reversed orientation. 3, After reinnervation ( $50-90 \mathrm{~d}$ ), the former posterior ILT muscle, now in the normal position of the anterior ILT, was injected with the second tracer. The tracers were diamidino yellow $(D Y)$ or HRP/WGA-HRP; when the first tracer was DY, the second tracer was WGA-HRP. When the first tracer was WGA-HRP, the second tracer was DY.

nervation, we chose the former approach.

The results of these double-label experiments are shown in Table 2. The results of 1 experiment in which fewer than 10 cells were labeled with HRP-perhaps indicative of poor reinnervation, inadequate HRP injection, or reaction (see below)have been excluded. For the other experiments, the mean number of HRP-labeled cells was $35 \pm 6$. Overall, $69 \pm 9 \%(n=8)$ of these neurons labeled with HRP after transplantation were also labeled with DY. Thus, the majority of individual motoneurons appeared to reinnervate their original target muscle selectively. Far fewer neurons $(41 \pm 5 \% ; p<0.01)$ were doubly labeled in 6 control experiments, in which the second tracer was injected into the adjacent anterior ILT instead. This last value should represent ncurons that reinnervated the muscle occupying the normal position of their target, rather than their original target muscle itself; thus, a minority of the neurons reinnervating a muscle are members of an inappropriate motor pool. Some, but not all, of these doubly labeled cells can probably be accounted for by the passage of dye from the injected muscle to its neighbors (or perhaps even between axons in nerves or neurons within the spinal cord; see the Discussion): in another class of control experiments on unoperated animals, we doubly labeled an appreciable number of neurons $(15 \pm 6 \%, n=9)$ when we injected DY into the posterior ILT and HRP into the anterior ILT. Direct microscopic observation of anterior and posterior ILT muscles, as well as iliofibularis, at the end of every experiment verified some apparently unavoidable cross-labeling of muscles. However, in only 1 case was the degree of muscle cross-labeling considered unacceptable (the labeling of noninjected muscles equaled that of the injected muscle) and excluded. Usually such cross-labeling was relatively minor.

Combined HRP/DY labeling. During the course of the experiments, it became apparent that DY might label more than just those neurons that innervated the injected muscle. Indeed, 2-3 months after DY injection into posterior ILT, the number of labeled motoneurons was significantly greater $(89 \pm 13, n=$ 14) than after $9-11 \mathrm{~d}(42 \pm 4, n=4 ; p<0.01)$ (Table 1). After long survival times, many cell nuclei were very faintly labeled; we excluded these from our counts. We suspected that the higher number of labeled cells after long survival times might reflect
Table 2. Incidence of doubly labeled cells

\begin{tabular}{lrrrrr} 
& \multicolumn{2}{c}{ Control (\%) } & & \multicolumn{2}{c}{ Experimental (\%) } \\
\cline { 2 - 3 } \cline { 5 - 6 } & $1^{a}$ & $2^{b}$ & & $1^{c}$ & $2^{d}$ \\
\hline Meane & 15 & 41 & 69 & 63 \\
SE & 6 & 5 & 9 & 6 \\
$n$ & 9 & 6 & 8 & 8
\end{tabular}

${ }^{a}$ In normal animals, the posterior ILT muscle was injected with DY and the anterior ILT with HRP.

${ }^{b}$ The second tracer (HRP in $5 / 6$ cases) was injected into the original anterior ILT muscle in animals that had already received the first tracer injection into posterior ILT (DY in $5 / 6$ cases) and in which the positions of the anterior and posterior ILT muscles had been reversed.

c First, DY was injected into the posterior ILT; after transplantation and reinnervation, HRP was injected into the original posterior ILT muscle, now in the normal position of the anterior ILT.

${ }^{d}$ Same as ${ }^{c}$ except that WGA-HRP, rather than DY, was injected first into the posterior ILT, and DY was used instead of HRP as the second tracer.

"Significance of differences between groups (Student's $t$ test) was as follows: $a$ and $b, p<0.01 ; b$ and $c, p<0.02 ; b$ and $d, p<0.02 ; c$ and $d, p<0.6$.

nonselective labeling of motoneurons that did not project to the injected muscle, or possibly the nuclei of glial cells (see the Discussion). We therefore repeated our experiment using WGAHRP as the initial label (Wan et al., 1982). Trial experiments showed that peroxidase activity was retained in a high proportion of the neurons initially labeled by injecting the posterior ILT with $10 \%$ WGA-HRP: by $50 \mathrm{~d}$ after injection, some 30 or so of the approximately 40 neurons usually labeled for short survival times were still clearly evident. The surprising longevity of the HRP label enabled us to conduct a reciprocal series of experiments, injecting WGA-HRP before surgery and DY after reinnervation, since the problems with DY were not evident shortly after DY injection. In 8 of 15 experiments, there were sufficient HRP- or DY-labeled cells detectable at the end of the experiment for analysis (cases in which fewer than 10 neurons were labeled with either tracer were excluded). In experiments satisfying these criteria, the mean number of HRP-labeled cells was $35 \pm 6$ (not significantly different from the number we labeled at short survival times; $p<0.2$ ); of these, $63 \pm 6 \%$, on average, were also labeled with DY (see Table 2; Fig. 5). This value agrees well with those obtained when DY was injected first $(69 \pm 9 \%)$, confirming our initial estimate of double-labeling. Because of this close agreement, we have not carried out reciprocal control experiments (as in Table 2, column 2), since it appears that spurious cell labeling by DY in long-term experiments did not contribute significantly to double-labeling.

\section{Analysis of axon reflexes}

A possible basis for the finding of doubly labeled motoneurons in control experiments might be that some motoneurons innervate both anterior and posterior ILT muscles. This could come about after motor axon regeneration and reinnervation of transplanted muscle (Stephenson, 1979), or perhaps even in normal animals. Thus, for example, the doubly labeled neurons we found in unoperated animals that received DY injections into posterior ILT and HRP injections into anterior ILT might represent neurons that branched to supply innervation to both anterior and posterior ILT muscles. We investigated this possibility by electrophysiologically testing for axon reflexes (Langley and Anderson, 1894; Stephenson, 1979) and for axons that passed from one muscle to the other, innervating both.

We first studied 10 hindlimbs from 6 unoperated axolotls. 

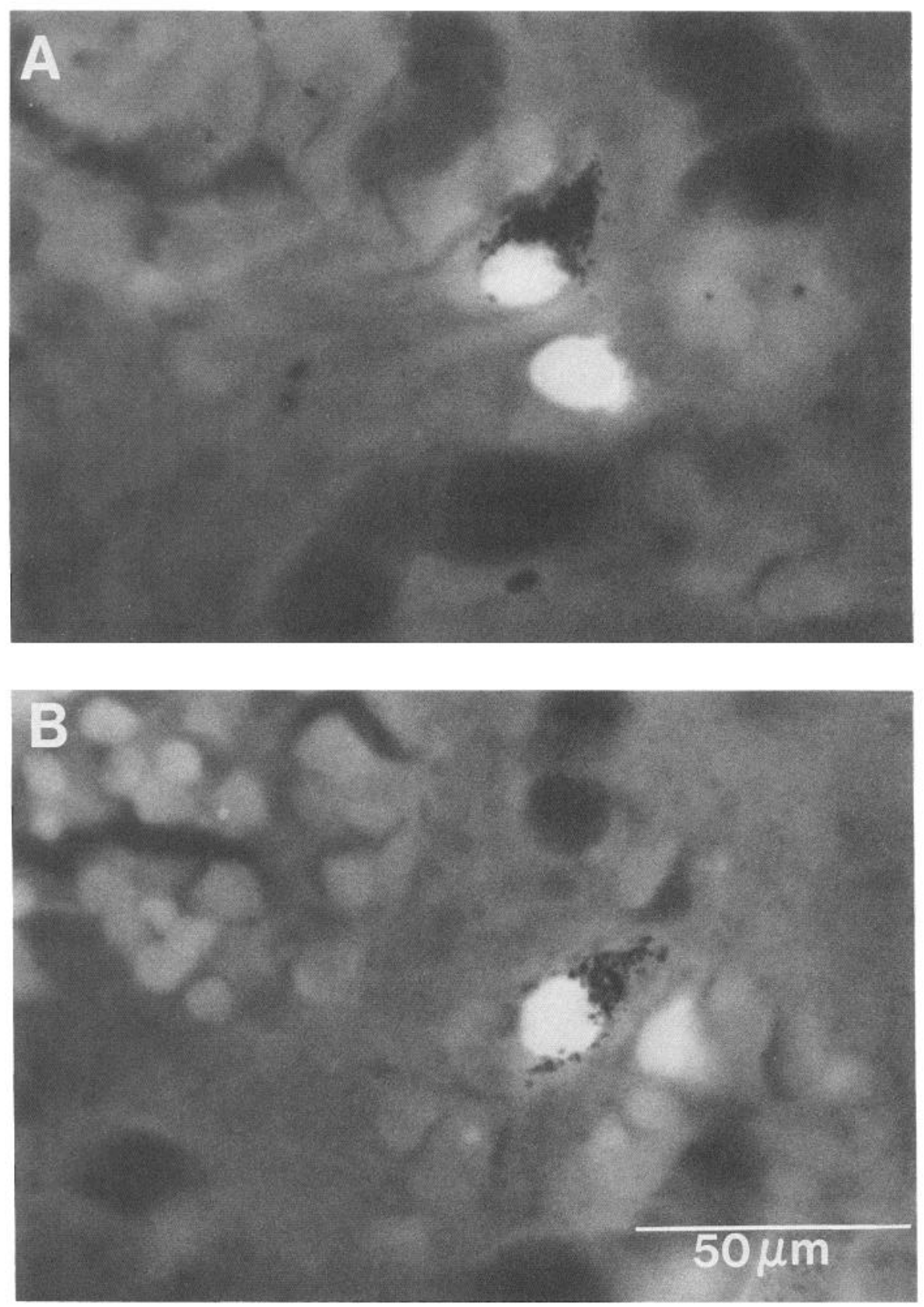

Figure 4. Photomicrographs of cells labeled with DY and HRP. $A$, Two DYlabeled cells, with brightly fluorescent nuclei, in the ventral horn of the spinal cord $82 \mathrm{~d}$ after injecting DY into the posterior ILT. One of the DY-labeled cells is also labeled with HRP, which was injected into the posterior ILT 67 $\mathrm{d}$ after exchanging the positions of the anterior and posterior muscles. $B, A$ single cell from the same spinal cord as in $A$, labeled with both DY and HRP. Both photomicrographs were obtained under simultaneous epi- and transillumination. Scale bar applies to both $A$ and $B$.
We usually tested 4 of the several nerves innervating each ILT muscle; thus, for each limb, a total of 16 combinations were possible. We monitored the fidelity of our recording system throughout these experiments by individually stimulating the spinal nerves that innervate the hindlimb and recording the action potentials in muscle nerves. The minimum response we could reliably detect with our recording system was about 10 $\mu \mathrm{V}$ in amplitude; single-unit responses could usually be discerned by varying stimulus intensity. In no case did stimulation of the proximal cut end of a posterior ILT muscle nerve initiate a response in the proximal cut end of an anterior ILT muscle nerve, nor vice versa. Thus, in agreement with Stephenson (1979), we conclude that few, if any, axon reflexes exist between different muscles in normal axolotls.

We also searched for axon reflexes between anterior and posterior ILT muscles in 9 animals that had undergone muscle transplantation 43-57 d earlier. Not a single axon reflex was observed between the nerves to 18 pairs of anterior and posterior ILT muscles. In 2 other animals (4 limbs), we left 1 of the ILT muscles in each limb intact. We then stimulated the proximal end of the 4 nerve branches that had supplied innervation to the excised ILT muscle, while we recorded extracellularly from the intact muscle with a suction electrode. This was done to further increase the likelihood that we would detect an axon reflex if it occurred-since each axon should innervate several muscle fibers, recordings obtained from an intact muscle ought to be more sensitive than those made from nerves. In 2 additional animals (4 limbs), both ILT muscles were left intact, and nerve trunks that provided innervation to either of the 2 muscles were exposed and cut, and their distal ends stimulated. Such stimulation always evoked action potentials in the muscle clearly supplied by the nerve, and we recorded these extracellularly. 
Figure 5. Photomicrograph of a neuron in the ventral horn of the axolotl spinal cord labeled with HRP and DY. This cell was labeled with HRP by injecting WGA-HRP into the posterior ILT muscle. After $63 \mathrm{~d}$, following the uptake of HRP, the exchange of anterior and posterior ILT muscles, and their reinnervation, DY was injected into the posterior ILT in its new position and subsequently labeled the nucleus of this motoneuron.

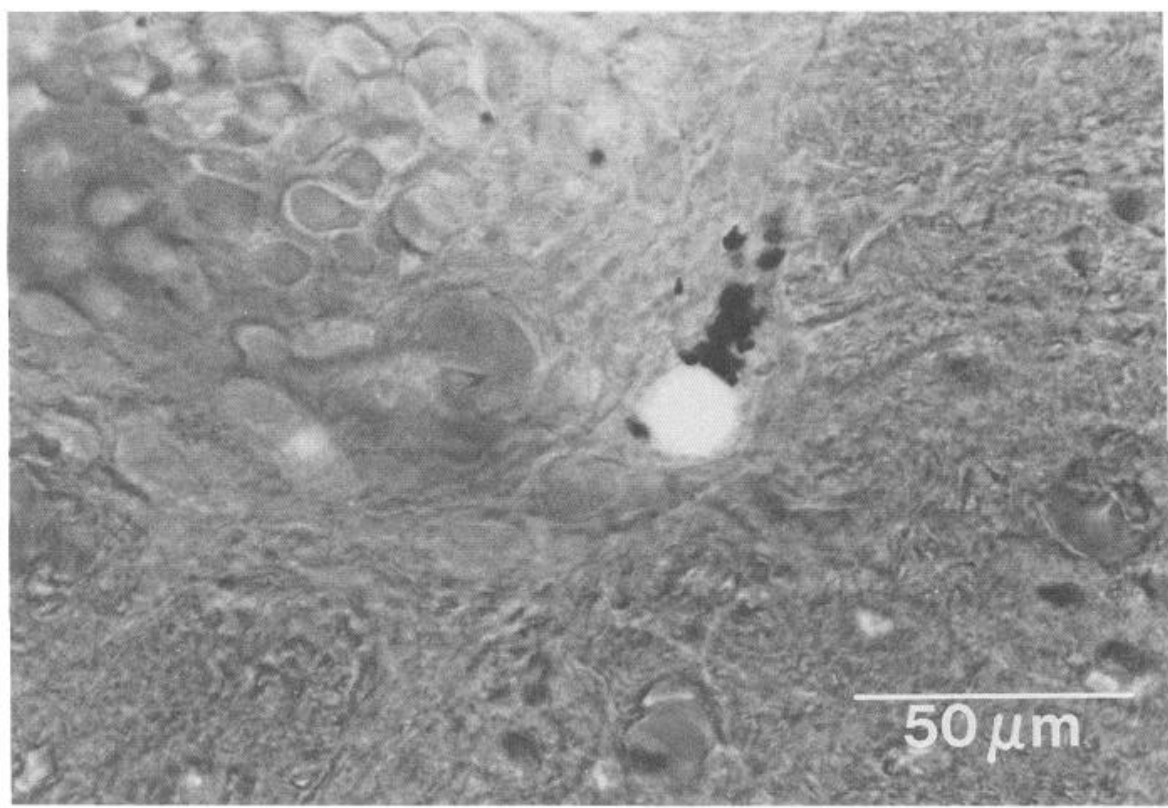

However, responses were never recorded in the adjacent muscle. Taken together, these results suggest that the axons that reinnervate a particular muscle do so exclusively: no cases of an axon providing innervation to more than 1 muscle were ever observed.

\section{Discussion}

These experiments address a long-standing question regarding the selectivity of reinnervation of limb muscles in urodele amphibia. It is generally believed, though it has not been directly proved, that motoneurons in axolotls selectively reinnervate their original target muscles after denervation (Grimm, 1971; Stephenson, 1979; Landmesser, 1980; Mark, 1980; Grinnell and Herrera, 1981; Holder et al., 1982, 1984). Most of the evidence for this conclusion has come from either behavioral observations of muscle function after reinnervation (see, for example, Detwiler, 1920; Weiss, 1937) or studies involving spinal nerve stimulation and intracellular recording (Cass and Mark, 1975; Bennett and Raftos, 1977; Dennis and Yip, 1978; Wigston, 1986). Considering the problems of interpreting behavioral data, and the inherent imprecision in identifying neuronal identity on the sole basis of the ventral root in which their axons exit the spinal cord, more rigorous approaches to examining selectivity have been needed.

Our approach was to use a long-term retrograde motoneuronlabeling technique, since it offered the possibility of identifying individual labeled neurons both before and after axotomy. We focused on 1 of 3 mechanism likely to contribute to the establishment of specificity, the selectivity of synapse formation itself, rather than axon guidance or synapse elimination, by transplanting muscles to novel positions and determining the degree to which they were reinnervated by members of their original motoneuron pool. Our results suggest that individual motoneurons selectively reinnervate their original muscles, even when these are in altered positions.

Motor pools in axolotls have been studied previously using electrophysiological methods (Székely and Czéh, 1967). Results obtained using this approach suggested that it would not be possible to identify motoneurons from their position in the sni- nal cord since neurons innervating adjacent or related muscles are extensively intermingled. This impression was confirmed by our own studies of the motor pools innervating anterior ILT, posterior ILT, and iliofibularis: using retrograde transport of HRP, we found extensive overlap between the pools. On the other hand, Stephens and Holder (1985) concluded from their studies on the innervation of axolotl limb muscles that retrograde HRP labeling could be used to assign motoneurons to their respective motor pools. It should be possible to distinguish flexor and extensor motoneurons on the basis of their position in the ventral horn of the spinal cord, since neurons that innervate flexor muscles tend to occupy relatively dorsal and lateral positions, whereas those innervating extensors are slightly more ventral (Stephens and Holder, 1985; D. J. Wigston and P. R. Kennedy, unpublished observations). Furthermore, since, on average, the motoneurons that innervate proximal limb muscles are located in more rostral regions of the spinal cord than those that innervate distal muscles, it is possible to judge, for some motoneurons, whether they normally belong to proximal or distal limb muscles (Stephens and Holder, 1985). This permits a limited assessment of the specificity of reinnervation of axolotl muscles (see Stephens and Holder, 1987). However, for the majority of neurons it is not feasible to identify them only by considering the position they occupy in the spinal cord. In our view, considering both the motor pool distributions found by Stephens and Holder (1985) and our own, the majority of axolotl motoneurons cannot be allocated unambiguously to a single motor pool solely on the basis of position. In any event, we found a wider distribution of motoneurons innervating either the anterior ILT or posterior ILT muscle, equivalent to the neurons that together innervate the entire ILT muscle, as studied by Stephens and Holder (1985). On one hand, we estimate the number of neurons innervating the combined ILT muscles to be greater than that reported by Stephens and Holder (1985) $(\approx 70 \mathrm{vs} \approx 25$ ). On the other, we find many neurons between ventral roots 16 and 17 that innervate either the anterior or posterior ILT (Fig. 2), whereas they found none. In view of our observation that electrical stimulation of spinal nerve 17 produces synaptic potentials in most muscle fibers in posterior ILT 
(Wigston, 1986), their result is somewhat surprising. Thus, even though we probably injected more HRP than they did, it is unlikely that this would account for our differences regarding motor pool distribution since our electrophysiological studies (Wigston, 1986) are consistent with our retrograde-labeling experiments.

In view of the limitations of conventional retrograde labeling, we took an alternative approach to identifying neurons after reinnervation, suggested by the persistence of fluorescent dyes within living cells for long periods (Bentivoglio et al., 1980; O'Leary et al., 1981; Sawchenko and Swanson, 1981; Cavada et al., 1984; Hendry et al., 1986). This persistence allowed us to label a motor pool with a stable marker; perform surgery, such as transplanting muscles; and then label the neurons reinnervating a particular muscle with a second tracer. Using 2 versions of this approach, which combined the fluorescent dye diamidino yellow with HRP histochemistry, we have reexamined the selectivity of the reinnervation of transplanted hindlimb muscles.

The double retrograde label approach we used had some inherent disadvantages. First, dye or HRP can spread between the injected and nearby muscles, since axolotl muscles are small and not well separated. Thus, motoneurons innervating muscles adjacent to the one injected can be labeled accidentally. This problem was particularly severe in smaller animals $(<12 \mathrm{~cm})$, where muscles are poorly ensheathed. We tried to reduce the amount of tracer spreading by using fully grown animals when available (mean, $16 \mathrm{~cm}$ ) and minimizing the amount of tracer injected. This was difficult, since axolotl muscles have multiple endplate zones and, therefore, completely labeling a muscle's motor pool necessitated injecting tracer at multiple sites throughout the muscle. We routinely monitored the degree of tracer crossover by examining all 3 muscles (anterior and posterior ILT and iliofibularis) after each experiment; in spite of all precautions, there was often evidence of some leakage between muscles. We attempted to quantify the degree of doublelabeling that might result from such crossover in control experiments on unoperated animals in which we injected the tracers simultaneously into neighboring muscles (see Table 2).

A second disadvantage of our technique was that the number of cells labeled with DY increased with time after injection; this may have caused us to overestimate the selectivity of reinnervation. 'The spurious labeling could not have been a result of continued uptake from the injected muscle because we had replaced the anterior ILT and the injected posterior ILT on the left side with a pair of unlabeled ILT muscles from the right side. The 2 most likely possibilities are that DY was somehow transferred between axons within nerves en route to the spinal cord, or that once in the cell bodies of neurons, DY can either escape and be taken up by neighboring cells (see Huisman et al., 1983) or be transferred directly to adjacent neurons or glia. At present we cannot distinguish between these alternatives.

Our solution to this adverse feature of DY was to reverse the order of tracer injection. HRP conjugated with WGA seemed to work surprisingly well as a long-term neuronal label and was still detectable $72 \mathrm{~d}$ after injection. Furthermore, the problems with spurious DY labeling were not apparent within short periods after injection (see Table 1). There was some loss of peroxidase activity, however, after long survival times; overall, fewer cells remained well labeled after long periods following injection. In 1 case, no HRP-labeled cells could be found. This introduced a different complication to the interpretation of our results: we may not have been revealing the total number of original posterior ILT motoneurons. However, such an error would underestimate the selectivity of reinnervation, since fewer DY-labeled neurons would then be expected to have HRP labeling as well. Thus, our estimate of $63 \%$ double-labeling in this set of experiments may in fact be lower than the proper value. Nevertheless, the close agreement between our results when using DY for either long- or short-term labeling suggests that spurious labeling did not contribute substantially to the number of doubly labeled neurons observed.

The interpretation that our results are indicative of selectivity must be tempered with a consideration of our control experiments (Table 2, column 2). These indicated that approximately $40 \%$ of the neurons that reinnervated transplanted anterior ILT muscles were not members of that muscle's original motor pool. The likelihood that most, if not all, of these doubly labeled cells were indeed posterior ILT motoneurons does not necessarily indicate a hierarchical preference for different motoneurons; the only neurons available to reinnervate transplanted anterior ILT muscles might have been anterior or posterior ILT motoneurons, since these were the only motor axons we severed. Unfortunately, our experiments address only 2 of several likely explanations for the rather high incidence of doubly labeled cells in these control experiments. First, the lack of axon reflexes in our electrophysiological studies suggests that no motoneurons innervate more than 1 muscle. It is conceivable, however, that at even earlier stages of reinnervation, axon reflexes may indeed have been present, but were eliminated before the times we looked. This is unlikely in view of our earlier work, which showed no significant rearrangement of initial motoneuron projection patterns after denervation and transplantation procedures identical to those employed here. Thus, the doubly labeled neurons in control experiments were probably not posterior ILT neurons that reinnervated both anterior and posterior ILT muscles. Second, the spurious labeling with DY in long-term experiments suggests that the double-labeling of cells in control experiments might be a result of the inadvertent labeling of anterior ILT motoncurons after injecting posterior ILT with DY. While a certain amount of this cross-labeling probably does occur (see Table 2, column 1), it is probably not sufficient to account for the number of doubly labeled cells we observed. Furthermore, it would be surprising if such dye transfer could have selectively labeled such a high proportion of the neurons that reinnervated the target muscle, since these neurons represented a small fraclion of the motoneurons present at this level of the spinal cord. None of 3 further possibilities can be ruled out at present. First, the doubly labeled cells in these control experiments might have been neurons that indiscriminately reinnervated the injected muscle. Second, they might have been neurons whose axons were constrained in some way by growth or guidance factors to innervate muscle fibers in the normal position of their original target muscle. Since the anterior and posterior ILT muscles had been exchanged, original posterior ILT neurons innervating anterior ILT after transplantation would havc bcen innervating a muscle in the normal position of their native muscle. A third and intriguing possibility is that these doubly labeled neurons were posterior ILT neurons that were somehow acceptable to the anterior ILT muscle: since anterior and posterior ILT muscles contain similar proportions of different muscle fiber types (N. Holder, personal communication), it is possible that a subset of posterior ILT motoneurons found muscle fibers within anterior ILT that were acceptable. However, our previous finding 
that foreign neurons (autonomic preganglionic neurons) reinnervate skeletal muscles selectively on the basis of a correspondence between the rostrocaudal origin of the muscle and the rostrocaudal position of the neurons in the spinal cord (Wigston and Sanes, 1982, 1985) suggests a different interpretation. Since the rostrocaudal position of some posterior ILT neurons corresponds with that of some anterior ILT neurons, some other quality, related to their position in the rostrocaudal axis, might make certain posterior ILT motoneurons acceptable to anterior ILT muscles. This raises the question of whether, had we used muscles that differed more dramatically in position or function, we might have observed greater selectivity in their reinnervation.

The main significance of the present work is that it examines reinnervation with regard to individual neurons rather than general regions of the spinal cord or spinal nerves. This has not been the case in previous studies of the selectivity of reinnervation, except for a few notable instances. In one example, individual muscle nerves were transposed and the efficiency of neuromuscular transmission at individual synapses was assayed in different target muscles (Holder et al., 1982). In another, sensory axons innervating particular muscles were shown to reinnervate preferentially motoneurons innervating the same muscle (Sah and Frank, 1984). Finally, selective reinnervation of targets by identified neurons has been documented in more simple invertebrate nervous systems (see, for example, Young, 1972; Wallace et al., 1977) and in tissue culture (Ready and Nicholls, 1979; Bodmer et al., 1984). Our own experiments with vertebrate motoneurons complement previous electrophysiological experiments on the same or similar systems, which have shown that transplanted muscles are reinnervated selectively by axons arising from appropriate spinal segments (Wigston, 1986; see also Wigston and Sanes, 1982, 1985). We have now shown that the most likely explanation for the selective reinnervation of axolotl muscles is that individual motoneurons have a good probability of reinnervating their original muscle despite its translocation to a new site. Thus, there must be a mechanism for the selective control of synapse formation that favors synaptic union between a particular motoneuron and specific target cells. This mechanism apparently operates in concert with, but independently of, mechanisms that control axonal guidance, since our experiments were designed to make the choice of proper pathways unnecessary for regenerating axons in relocating their original muscle. While the anatomical arrangement of the nerve branches that supply anterior and posterior ILT in the axolotl is such that both muscles are easily accessible to the cut cnds of axons that previously innervated either muscle, we cannot strictly rule out the possibility of short-range, musclespecific tropic signals, or the attraction of severed axons towards, and guidance down, the distal stumps of their original nerve. However, unpublished results from this laboratory suggest that anterior and posterior ILT muscles can be distinguished from one another even after transplantation into the forelimb and reinnervation by foreign, brachial motoneurons. These and previous findings (Wigston and Sanes, 1982, 1985; Laskowski and Sanes, 1987) indicate that one factor contributing to the identity of pre- and postsynaptic elements might be some aspect of their position in the rostrocaudal axis. We favor the view that the selectivity we observe depends on a system of recognition cuesperhaps molecules associated with the surfaces of muscle fibers, which are used by the growth cones of regenerating axons when choosing their synaptic partners.

\section{References}

Abercrombie, M. (1946) Estimation of nuclear population from microtome sections. Anat. Rec. 94: 239-247.

Bennett, M. R., and J. Raftos (1977) The formation and regression of synapses during the re-innervation of axolotl striated muscle. J. Physiol. (Lond.) 265: 261-295.

Bentivoglio, M., H. G. J. M. Kuypers, C. E. Catsman-Berrevoets, H. Loewe, and O. Dann (1980) Two new fluorescent retrograde neuronal tracers which are transported over long distances. Neurosci. Lett. 18: 25-30.

Bodmer, R., D. Dagan, and I. B. Levitan (1984) Chemical and electrotonic connections between Aplysia neurons in primary culture. $\mathrm{J}$. Neurosci. 4: 228-233.

Brushart, T. M., and M.-M. Mesulam (1980) Alteration in connections between muscle and anterior horn motoneurons after peripheral nerve repair. Science 208: 603-605.

Cass, D. T., and R. F. Mark (1975) Re-innervation of axolotl limbs I. Motor nerves. Proc. R. Soc. Lond. [Biol.] 190: 45-58.

Cavada, C., A. M. Huisman, and H. G. J. M. Kuypers (1984) Retrograde double labeling of neurons: The combined use of horseradish peroxidase and diamidino yellow dihydrochloride (DY. $2 \mathrm{HCl}$ ) compared with True Blue and $\mathrm{DY} .2 \mathrm{HCl}$ in rat descending brainstem pathways. Brain Res. 308: 123-136.

Dennis, M. J., and J. W. Yip (1978) Formation and elimination of foreign synapses on adult salamander muscle. J. Physiol. (Lond.) 274: 299-310.

Detwiler, S. R. (1920) Experiments on the transplantation of limbs in Amblystoma. The formation of nerve plexuses and the function of the limbs. J. Exp. Zool. 31: 117-169.

Grimm, L. M. (1971) An evaluation of myotypic respecification in axolotls. J. Exp. Zool. 178: 479-496.

Grinnell, A. D., and A. A. Herrera (1981) Specificity and plasticity of neuromuscular connections: Long term regulation of motoneuron function. Prog. Neurobiol. 17: 203-282.

Hendry, I. A., C. E. Hill, and D. J. Watters (1986) Long-term retention of fast blue in sympathetic neurones after axotomy and regenerationdemonstration of incorrect connections. Brain Res. 376: 292-298.

Holder, N., J. Mills, and D. A. Tonge (1982) Selective reinnervation of skeletal muscle in the newt Triturus cristatus. J. Physiol. (Lond.) 326: 371-384.

Holder, N., D. A. Tonge, and P. Jesani (1984) Directed regrowth of axons from a misrouted nerve to their correct muscles in the limb of the adult newt. Proc. R. Soc. Lond. [Biol.] 222: 477-489.

Hollyday, M. (1980) Organization of motor pools in the chick lumbar lateral motor column. J. Comp. Neurol. 194: 143-170.

Hollyday, M., V. Hamburger, and J. M. G. Faris (1977) Localization of motor neuron pools supplying identified muscles in normal and supernumerary legs of chick embryo. Proc. Natl. Acad. Sci. USA 74: 3582-3586.

Huisman, A. M., H. G. J. M. Kuypers, F. Condé, and K. Keizer (1983) Collaterals of rubrospinal neurons to the cerebellum in rat. A retrograde fluorescent double labeling study. Brain Res. 264: 181-196.

Lamb, A. H. (1976) The projection patterns of the ventral horn to the hind limb during development. Dev. Biol. 54: 82-99.

Lance-Jones, C., and L. Landmesser (1980) Motoneurone projection patterns in the chick hind limb following early partial reversals of the spinal cord. J. Physiol. (Lond.) 302: 581-602.

Landmesser, L. (1978) The distribution of motoneurones supplying chick hind limb muscles. J. Physiol. (Lond.) 284: 371-389.

Landmesser, L. (1980) The generation of neuromuscular specificity. Annu. Rev. Neurosci. 3: 279-302.

Langley, J. N., and H. K. Anderson (1894) On reflex action from sympathetic ganglia. J. Physiol. (Lond.) 16: 410-440.

Laskowski, M. B., and J. R. Sanes (1987) Topographic mapping of motor pools onto skeletal muscles. J. Neurosci. 7: 252-260.

Lehouelleur, J., and A. Chatelain (1974) Analysis of electrical responses of newt skeletal muscle fibres in response to direct and indircct stimulation. J. Physiol. (Paris) 68: 615-632.

Mark, R. F. (1980) Synaptic repression at neuromuscular junctions. Physiol. Rev. 60: 355-395.

Mesulam, M.-M. (1978) Tetramethyl benzidine for horseradish peroxidase neurohistochemistry: A non-carcinogenic blue reaction-product with superior sensitivity for visualizing neural afferents and efferents. J. Histochem. Cytochem. 26: 106-117. 
O'Leary, D. D. M., B. B. Stanfield, and W. M. Cowan (1981) Evidence that the early postnatal restriction of the cells of origin of the callosal projection is due to the elimination of axonal collaterals rather than to the death of neurons. Dev. Brain Res. $1: 607-617$.

Ready, D. F., and J. G. Nicholls (1979) Identified neurones isolated from leech CNS make selective connexions in culture. Nature 281 : 67-69.

Sah, D. W. Y., and E. Frank (1984) Regeneration of sensory-motor synapses in the spinal cord of the bullfrog. J. Neurosci. 4: 2784-2791.

Sawchenko, P. E., and L. W. Swanson (1981) A method for tracing biochemically defined pathways in the central nervous system using combined fluorescence retrograde transport and immunocytochemical techniques. Brain Res. 210: 31-51.

Slack, J. R., and J. R. Docherty (1978) The pattern of innervation of a polyneural muscle: Axolotl iliotibialis. Cell Tissue Res. 186: 171180.

Stephens, N., and N. Holder (1985) A horseradish peroxidase study of motorneuron pools of the forelimb and hindlimb musculature of the axolotl. Proc. R. Soc. Lond. [Biol.] 224: 325-339.

Stephens, N., and N. Holder (1987) Reformation of the pattern of neuromuscular connections in the regenerated axolotl hindlimb. Dcvelopment 99: 221-230.

Stephenson, R. S. (1979) Axon reflexes in axolotl limbs: Evidence that branched motor axons reinnervate muscles selectively. Exp. Neurol 64: 174-189.

Székely, G., and G. Czéh (1967) Localization of motoneurones in the limb moving spinal cord of Ambystoma. Acta Physiol. Acad. Sci. Hung. 32: 3-18.
Wallace, B. G., M. N. Adal, and J. G. Nicholls (1977) Sprouting and regeneration of synaptic connexions by sensory neurones in leech ganglia maintained in culture. Proc. R. Soc. Lond. [Biol.] 199: 567585.

Wan, X.-C. S., J. Q. Trojanowski, and J. O. Gonatas (1982) Cholera toxin and wheat germ agglutinin conjugates as neuroanatomical probes: Their uptakc and clcarance, transganglionic and retrograde transport and sensitivity. Brain Res. 243: 215-224.

Weiss, P. (1937) Further experimental investigations on the phenomenon of homologous response in transplanted amphibian limbs I. Functional observations. J. Comp. Neurol. 66: 181-209.

Wigston, D. J. (1986) Selective innervation of transplanted limb muscles by regenerating motor axons in the axolotl. J. Neurosci. 6: 27572763.

Wigston, D. J., and P. R. Kennedy (1986) Selective reinnervation of axolotl limb muscles by their original motoneurons. Soc. Neurosci. Abstr. 12: 541 .

Wigston, D. J., and J. R. Sanes (1982) Selective reinnervation of adult mammalian muscle by axons from different segmental levels. Nature 299: 464-467.

Wigston, D. J., and J. R. Sancs (1985) Sclective reinnervation of intercostal muscles transplanted from different segmental levels to a common site. J. Neurosci. 5: 1208-1221.

Young, D. (1972) Specific re-innervation of limbs transplanted between segments in the cockroach, Periplaneta americana. J. Exp. Biol. 57: $305-316$ 